\title{
Is overwork weakness relevant in Charcot-Marie-Tooth disease?
}

\author{
G Piscosquito, ${ }^{1}$ M M Reilly, ${ }^{2}$ A Schenone, ${ }^{3}$ G M Fabrizi, ${ }^{4}$ T Cavallaro, ${ }^{4}$ L Santoro, ${ }^{5}$ \\ G Vita, ${ }^{6}$ A Quattrone, ${ }^{7}$ L Padua, ${ }^{8}$ F Gemignani, ${ }^{9}$ F Visioli, ${ }^{10,11}$ M Laurà, ${ }^{2}$ \\ D Calabrese, ${ }^{1}$ R A C Hughes, ${ }^{2}$ D Radice, ${ }^{12}$ A Solari, ${ }^{1}$ D Pareyson, ${ }^{1}$ \\ for the CMT-TRIAAL \& CMT-TRAUK Group
}

For numbered affiliations see end of article.

\section{Correspondence to Dr Davide Pareyson, Clinic of Central and Peripheral Degenerative Neuropathies Unit, Department of Clinical Neurosciences-IRCCS Foundation, C. Besta Neurological Institute, via Celoria 11, Milan 20133, Italy; davide.pareyson@istituto-besta.}

Received 10 January 2014 Revised 25 February 2014 Accepted 26 February 2014 Published Online First 21 March 2014

\section{SLinked}

- http://dx.doi.org/10.1136/ jnnp-2014-307924

CrossMark

\section{ABSTRACT}

Background In overwork weakness (OW), muscles are increasingly weakened by exercise, work or daily activities. Although it is a well-established phenomenon in several neuromuscular disorders, it is debated whether it occurs in Charcot-Marie-Tooth disease (CMT).

Dominant limb muscles undergo a heavier overload than non-dominant and therefore if OW occurs we would expect them to become weaker. Four previous studies, comparing dominant and non-dominant hand strength in CMT series employing manual testing or myometry, gave contradictory results. Moreover, none of them examined the behaviour of lower limb muscles.

Methods We tested the OW hypothesis in 271 CMT1A adult patients by comparing bilateral intrinsic hand and leg muscle strength with manual testing as well as manual dexterity.

Results We found no significant difference between sides for the strength of first dorsal interosseous, abductor pollicis brevis, anterior tibialis and triceps surae. Dominant side muscles did not become weaker than non-dominant with increasing age and disease severity (assessed with the CMT Neuropathy Score); in fact, the dominant triceps surae was slightly stronger than the non-dominant with increasing age and disease severity.

Discussion Our data does not support the OW hypothesis and the consequent harmful effect of exercise in patients with CMT1A. Physical activity should be encouraged, and rehabilitation remains the most effective treatment for CMT patients.

\section{INTRODUCTION}

Overwork weakness (OW) is characterised by a progressive muscular weakening due to exercise, work or daily activities. It has been demonstrated in several neuromuscular diseases including postpolio syndrome, ${ }^{1}$ facio-scapulo-humeral and Duchenne muscular dystrophies, ${ }^{2}{ }^{3}$ and amyotrophic lateral sclerosis. ${ }^{4}$ In these disorders, muscle overload increases disease progression. It is matter for debate whether OW plays a role also in Charcot-Marie-Tooth disease (CMT). The answer is of utmost importance because it will greatly influence the advice to perform physical activity and rehabilitation rather than to spare involved muscles for fear of further worsening. If OW plays a relevant role in CMT, we would expect a significant difference in hand strength (HS) in favour of the non-dominant hand $(\mathrm{NDH})$, because dominant hand $(\mathrm{DH})$ muscles undergo a heavier overload.

Vinci and coauthors found the DH muscles to be weaker in about $66 \%$ of evaluated muscles in a series of 106 patients with different types of CMT (80 demyelinating CMT1 and 26 axonal CMT2 forms) by evaluating muscle research council scores on a 14-point scale. Therefore, they concluded that OW produces additional weakness and may be a cause of disease progression. ${ }^{5}$ By contrast, Van Pomeren et al tested a series of 28 CMT patients (13 CMT1 and 15 CMT2) by using the conventional 6-point MRC scale and also the Rotterdam Intrinsic Hand Myometer, which allows measuring intrinsic hand muscle strength. There was no finding in favour of the OW hypothesis; rather, in CMT2 patients the key-grip test showed the $\mathrm{DH}$ to be stronger than the $\mathrm{NDH} .{ }^{6}$ By using a digital handgrip dynamometer, Videler and colleagues found no significant differences between sides for grip, 2-point, tripod and lateral pinch strength in a series of 49 patients with CMT type 1A (the most common CMT type). However, in the more severely affected subgroup (22 patients), pinch strength was significantly lower in the DH, which might fit the OW hypothesis. ${ }^{7}$ Similarly, Arthur-Farraj and coauthors evaluated a series of 43 patients with the X-linked CMT type (CMTX1) and found that in patients with MRC grade less than 4-, the abductor pollicis brevis (APB) and first dorsal interosseous (FDI) muscles of the NDH were stronger than that of the $\mathrm{DH}$, a finding which again might be in favour of OW. They also showed a relative reduction of the amplitude of the median nerve compound muscle action potential (CMAP) in the DH as compared with the $\mathrm{NDH}$, whereas no differences between sides were found for median and ulnar motor nerve conduction velocities and ulnar nerve CMAP amplitude. ${ }^{8}$ None of the previous studies tested the lower limbs, where the effect of dominance is still debated. ${ }^{9}$ In order to verify the OW hypothesis in our population of 271 adult CMT1A patients enrolled in the ascorbic acid trial, we compared intrinsic hand and leg muscle strength in search of possible strength differences between sides. ${ }^{10}$ We also tested manual dexterity because Videler and coauthors have shown that tripod pinch and thumb opposition strength are major determinants of manual dexterity in 
CMT1A and proposed focused exercise programmes to improve manual dexterity. ${ }^{11}$

\section{MATERIAL AND METHODS}

We retrospectively reviewed data on the 271 CMT1A patients (163 women and 108 men), aged 18-70 years (mean 42.5 years), recruited in the Italian/UK trial of ascorbic acid (CMT-TRIAAL/CMT-TRAUK; baseline visit). ${ }^{10}$ Other causes of hand or foot weakness were ruled out. We assessed muscle strength by manual testing using the classical 6-point MRC scale (range 0-5). We tested bilaterally the FDI, APB, anterior tibialis (AT) and triceps surae (TS) muscles, as distal muscles are the weakest in CMT. For each limb, a combined value was obtained by averaging MRC scores of the couples of muscles, FDI and APB for the hand and AT and TS for the foot, thus obtaining for each side a HS and foot strength (FS) score, respectively. Manual dexterity was also assessed using the 9-hole peg test and expressed in seconds (average of two tests for each side after two test trials). We determined whether muscles were stronger on dominant or non-dominant side and computed the difference between them ( $\Delta=\mathrm{D}-\mathrm{ND})$. We defined 'asymmetry' a $\geq 1$-point difference in MRC score between the two sides. It was considered positive if the dominant side was stronger, negative if the dominant side was weaker. Hand/foot dominance assessment: the patient indicated his/her preferred hand/leg; in case of uncertainty, the patient was asked to think about writing, holding a glass, walking up a stair, biking and playing soccer and the dominant side determined accordingly. Ethics committee approval was obtained from all involved centres; all patients gave written informed consent.

Categorical variables were summarised as counts and percentages and compared using the $\chi^{2}$ test or Fisher's exact test, as appropriate. Continuous variable were summarised as means and SDs; they were compared using the Wilcoxon ranksum test or the Kruskal-Wallis for comparisons involving classification variables with more than two levels. Normality assumption was checked with the Shapiro-Wilk normality test.

To assess the effect of patient age and disease severity, we categorised a priori age into four classes which were sufficiently balanced (18-30 years, 31-40 years, 41-50 years, 51-70 years) and CMT Neuropathy Score (CMTNS) values into three classes (mild, 0-10; moderate, 11-20; severe disease, 21-36).

Analyses were performed with SAS V.9.2 (Cary, North Carolina, USA). All statistical tests were two-tailed, and considered significant at the $\alpha$ level of 0.05 .

\section{RESULTS}

Strength differences between dominant and non-dominant sides

In all, 16 patients had left side dominance whereas 255 were right-handed.

MRC score means of all tested muscles (FDI, APB, AT, TS) are reported in table 1 . There were no significant differences between the sides for any muscle group. As expected, manual dexterity was significantly better on the dominant side (table 1).

\section{Symmetric or asymmetric weakness?}

HS was symmetric in the majority of CMT patients, both for FDI and APB: $87.4 \%$ of subjects had no difference in MRC score between sides, and asymmetry was observed only in $12.6 \%$ of patients (with DH stronger in $5.2 \%$ and weaker in $7.4 \%$ of the cases). Similarly, FS was symmetric in most cases (AT 81.1\%; TS 94.4\%) with non-significant differences between sides in asymmetric patients (table 2).
Table 1 Comparison of strength and manual dexterity in dominant and non-dominant muscles

\begin{tabular}{|c|c|c|c|c|c|}
\hline \multirow[b]{2}{*}{ Muscle strength (MRC) } & \multirow[b]{2}{*}{$\mathbf{N}$} & \multicolumn{4}{|l|}{ Mean \pm SD } \\
\hline & & D & ND & $\Delta$ & p Value \\
\hline FDI & 271 & $3.73 \pm 0.87$ & $3.74 \pm 0.96$ & $-0.01 \pm 0.38$ & 0.65 \\
\hline APB & 271 & $3.81 \pm 0.98$ & $3.83 \pm 1.00$ & $-0.02 \pm 0.39$ & 0.36 \\
\hline AT & $270 *$ & $3.40 \pm 1.37$ & $3.37 \pm 1.39$ & $0.03 \pm 0.58$ & 0.52 \\
\hline TS & $269+$ & $4.58 \pm 1.00$ & $4.55 \pm 1.03$ & $0.03 \pm 0.26$ & 0.10 \\
\hline HS & 271 & $3.77 \pm 0.85$ & $3.78 \pm 0.90$ & $-0.01 \pm 0.29$ & 0.39 \\
\hline FS & $269+$ & $3.99 \pm 1.06$ & $3.96 \pm 1.10$ & $0.03 \pm 0.32$ & 0.21 \\
\hline \multicolumn{6}{|l|}{ Manual dexterity } \\
\hline 9-HPT (s) & 271 & $23.2 \pm 6.8$ & $25.1 \pm 7.1$ & $-1.86 \pm 3.27$ & $<0.001$ \\
\hline \multicolumn{6}{|c|}{$\begin{array}{l}\text { Bold value indicates significant difference. } \\
\text { Average difference ( } \triangle=D-N D) \text { of strength and manual dexterity (9-HPT) between the } \\
\text { two sides is shown. Values indicate the mean and SD of all tested muscles. } \\
\text { *One missing value. } \\
\text { †Two missing values. } \\
\text { 9-HPT: 9-hole peg test; APB, abductor pollicis brevis; AT, anterior tibialis; D, dominant } \\
\text { side; FDI, first dorsal interosseous; FS, foot strength (average between AT and TS); } \\
\text { HS, hand strength (average between FDI and APB); N, number of tested patients; } \\
\text { ND, non-dominant side; TS, triceps surae. }\end{array}$} \\
\hline
\end{tabular}

\section{Effect of ageing on strength according to dominance}

For both sides, there was a significant decrease in strength with ageing for all tested muscles $(\mathrm{p}<0.001)$. However, there were no significant differences between the $\mathrm{DH}$ and $\mathrm{NDH}$ decreases for any age group. In the lower limbs, there were no differences between sides for AT strength according to age whereas there was a slight but significant difference for TS strength which was greater on the dominant side as compared with the nondominant in all age groups except for $41-50$ years (table 3 ).

\section{Disease severity and strength}

There was a significant decrease in strength on both sides for all tested muscles with increasing disease severity according to CMTNS values $(p<0.001)$. However, the DH did not become weaker than the NDH with increasing CMTNS values and the differences were not significant for any disease severity class. In lower limbs, there were no differences for AT strength, but the dominant TS became stronger than the non-dominant with increasing disease severity (table 4).

There was no effect of gender on differences in strength between sides and asymmetry (table 5).

\section{DISCUSSION}

The objective of this study was to determine if the OW hypothesis is true in CMT, as previous studies gave contradictory results

Table 2 Percentage of patients with symmetry and asymmetry of muscle strength

\begin{tabular}{lllllll}
\hline \multicolumn{7}{c}{ N (row \%) } \\
\cline { 3 - 5 } & & N & D stronger & Equal & ND stronger & p Value \\
\hline Hand & FDI & 271 & $14(5.2)$ & $237(87.4)$ & $20(7.4)$ & 0.38 \\
& APB & 271 & $14(5.2)$ & $237(87.4)$ & $20(7.4)$ & 0.38 \\
Foot & AT & $270^{*}$ & $27(10.0)$ & $219(81.1)$ & $24(8.9)$ & 0.67 \\
& TS & $269+$ & $11(4.1)$ & $254(94.4)$ & $4(1.5)$ & 0.07 \\
\hline * One missing value. & & & \\
†Two missing values. \\
APB, abductor pollicis brevis; AT, anterior tibialis; D, dominant side; FDI, first dorsal \\
interosseous; N, number of tested patients; ND, non-dominant side; TS, triceps surae.
\end{tabular}


Table 3 Differences in muscle strength between sides according to age

\begin{tabular}{|c|c|c|c|c|c|}
\hline \multirow[b]{2}{*}{ Age (years) } & \multirow[b]{2}{*}{$\mathrm{N}$} & \multicolumn{3}{|l|}{ Mean \pm SD } & \multirow[b]{2}{*}{$p$ Value for $\Delta^{*}$} \\
\hline & & D & ND & $\Delta$ & \\
\hline \multicolumn{6}{|l|}{ FDI } \\
\hline$\leq 30$ & 60 & $4.00 \pm 0.52$ & $4.05 \pm 0.59$ & $-0.05 \pm 0.29$ & 0.582 \\
\hline $31-40$ & 55 & $4.00 \pm 0.75$ & $4.04 \pm 0.79$ & $-0.04 \pm 0.33$ & \\
\hline $41-50$ & 68 & $3.76 \pm 0.82$ & $3.72 \pm 0.93$ & $0.04 \pm 0.37$ & \\
\hline $51-70$ & 88 & $3.34 \pm 1.04$ & $3.35 \pm 1.12$ & $-0.01 \pm 0.48$ & \\
\hline Overall & 271 & $3.72 \pm 0.87$ & $3.74 \pm 0.96$ & $-0.01 \pm 0.38$ & \\
\hline \multicolumn{6}{|l|}{ APB } \\
\hline$\leq 30$ & 60 & $4.12 \pm 0.67$ & $4.20 \pm 0.66$ & $-0.08 \pm 0.38$ & 0.298 \\
\hline $31-40$ & 55 & $4.00 \pm 0.88$ & $3.96 \pm 0.90$ & $0.04 \pm 0.33$ & \\
\hline $41-50$ & 68 & $3.90 \pm 0.88$ & $3.93 \pm 0.87$ & $-0.03 \pm 0.34$ & \\
\hline $51-70$ & 88 & $3.42 \pm 1.15$ & $3.43 \pm 1.19$ & $-0.01 \pm 0.47$ & \\
\hline Overall & 271 & $3.81 \pm 0.98$ & $3.83 \pm 1.00$ & $-0.02 \pm 0.39$ & \\
\hline \multicolumn{6}{|l|}{ AT } \\
\hline$\leq 30$ & 60 & $3.90 \pm 1.00$ & $3.90 \pm 0.93$ & $0.00 \pm 0.66$ & 0.309 \\
\hline $31-40$ & 55 & $3.89 \pm 0.98$ & $3.75 \pm 1.09$ & $0.15 \pm 0.52$ & \\
\hline $41-50$ & $67 \dagger$ & $3.30 \pm 1.35$ & $3.28 \pm 1.42$ & $0.01 \pm 0.44$ & \\
\hline $51-70$ & 88 & $2.83 \pm 1.57$ & $2.83 \pm 1.58$ & $0.00 \pm 0.64$ & \\
\hline Overall & 270 & $3.40 \pm 1.37$ & $3.37 \pm 1.39$ & $0.03 \pm 0.58$ & \\
\hline \multicolumn{6}{|l|}{ TS } \\
\hline$\leq 30$ & 60 & $4.83 \pm 0.49$ & $4.82 \pm 0.50$ & $0.02 \pm 0.13$ & 0.036 \\
\hline $31-40$ & $54 t$ & $4.78 \pm 0.63$ & $4.76 \pm 0.67$ & $0.02 \pm 0.24$ & \\
\hline $41-50$ & $67 t$ & $4.51 \pm 1.12$ & $4.54 \pm 1.11$ & $-0.03 \pm 0.17$ & \\
\hline $51-70$ & 88 & $4.34 \pm 1.25$ & $4.25 \pm 1.32$ & $0.09 \pm 0.36$ & \\
\hline Overall & 269 & $4.58 \pm 1.00$ & $4.55 \pm 1.03$ & $0.03 \pm 0.26$ & \\
\hline
\end{tabular}

Bold value indicates significant difference.

Average difference $(\Delta=\mathrm{D}-\mathrm{ND})$ of strength between $\mathrm{DH}$ and $\mathrm{NDH}$ is shown by age.

Values indicate the mean and SD of all tested muscles.

*Between age classes overall comparison by Kruskal-Wallis test.

tOne missing value.

APB, abductor pollicis brevis; $\mathrm{AT}$, anterior tibialis; $\mathrm{D}$, dominant side; $\mathrm{DH}$, dominant hand; FDI, first dorsal interosseous; $\mathrm{N}$, number of tested patients; ND, non-dominant side; NDH, non-dominant hand; TS, triceps surae.

Table 4 Differences in muscle strength between sides according to disease severity as assessed with the CMT Neuropathy Score (CMTNS)

\begin{tabular}{llrllll}
\hline & \multicolumn{5}{l}{ Mean \pm SD } & \\
\cline { 4 - 6 } & CMTNS & N & D & ND & \multicolumn{1}{c}{$\boldsymbol{~}$} & p Value for $\boldsymbol{\Delta}^{*}$ \\
\hline FDI & Mild & 55 & $4.15 \pm 0.40$ & $4.18 \pm 0.43$ & $-0.04 \pm 0.33$ & 0.634 \\
& Moderate & 187 & $3.80 \pm 0.72$ & $3.82 \pm 0.82$ & $-0.02 \pm 0.39$ & \\
& Severe & 25 & $2.24 \pm 1.20$ & $2.20 \pm 1.22$ & $0.04 \pm 0.35$ & \\
APB & Mild & 55 & $4.22 \pm 0.76$ & $4.31 \pm 0.77$ & $-0.09 \pm 0.40$ & 0.202 \\
& Moderate & 187 & $3.83 \pm 0.89$ & $3.84 \pm 0.87$ & $-0.01 \pm 0.37$ & \\
& Severe & 26 & $2.77 \pm 1.27$ & $2.77 \pm 1.42$ & $0.00 \pm 0.57$ & \\
AT & Mild & 55 & $4.45 \pm 0.69$ & $4.36 \pm 0.59$ & $0.09 \pm 0.40$ & 0.192 \\
& Moderate & 187 & $3.32 \pm 1.27$ & $3.31 \pm 1.32$ & $0.01 \pm 0.64$ & \\
& Severe & 25 & $1.76 \pm 1.33$ & $1.68 \pm 1.38$ & $0.08 \pm 0.49$ & \\
TS & Mild & 55 & $4.98 \pm 0.13$ & $4.98 \pm 0.13$ & $0.00 \pm 0.00$ & 0.011 \\
& Moderate & 186 & $4.64 \pm 0.85$ & $4.62 \pm 0.91$ & $0.02 \pm 0.27$ & \\
& Severe & 25 & $3.28 \pm 1.81$ & $3.12 \pm 1.72$ & $0.16 \pm 0.37$ & \\
\hline
\end{tabular}

Bold value indicates significant difference.

Average difference ( $\Delta=D-N D)$ of strength between DH and NDH according disease severity (as assessed by CMTNS) is shown. Three subgroups were considered: mild (0-10); moderate (11-20); severe (21-36).

*Between CMTNS levels overall comparison by Kruskal-Wallis test.

APB, abductor pollicis brevis; AT, anterior tibialis; CMT, Charcot-Marie-Tooth; D,

dominant side; $\mathrm{DH}$, dominant hand; $\mathrm{FDI}$, first dorsal interosseous; $\mathrm{N}$, number of tested

patients; ND, non-dominant side; NDH, non-dominant hand; TS, triceps surae.
Table 5 Side of the strongest muscle by sex

\begin{tabular}{|c|c|c|c|c|c|}
\hline \multirow[b]{2}{*}{ Sex } & \multirow[b]{2}{*}{$\mathrm{N}$} & \multicolumn{3}{|c|}{ N (row \%) } & \multirow[b]{2}{*}{ p Values* } \\
\hline & & ND & Equal & D & \\
\hline \multicolumn{6}{|l|}{ FDI } \\
\hline Male & $107 \dagger$ & $7(6.5)$ & 98 (91.6) & $2(1.9)$ & 0.124 \\
\hline Female & 163 & $12(7.4)$ & $139(85.3)$ & $2(7.4)$ & \\
\hline \multicolumn{6}{|l|}{ APB } \\
\hline Male & $107 \dagger$ & $7(6.5)$ & $94(87.0)$ & $7(6.5)$ & 0.716 \\
\hline Female & 163 & $12(7.4)$ & $144(88.3)$ & $7(4.3)$ & \\
\hline \multicolumn{6}{|l|}{ AT } \\
\hline Male & $107 \dagger$ & $10(9.4)$ & 85 (79.4) & $12(11.2)$ & 0.844 \\
\hline Female & 163 & $14(8.6)$ & $134(82.2)$ & $15(9.2)$ & \\
\hline \multicolumn{6}{|l|}{ TS } \\
\hline Male & $106 \ddagger$ & $1(0.9)$ & $103(97.2)$ & $2(1.9)$ & 0.317 \\
\hline Female & 163 & $3(1.8)$ & 151 (92.6) & $9(5.5)$ & \\
\hline
\end{tabular}

and were inconclusive. We tested muscle strength in our series of 271 CMT1A patients to see whether it was lower on the dominant side as compared with the non-dominant, which would indicate the occurrence of OW. Our study is the largest study conducted to date, tested a genetically homogeneous CMT population (CMT1A) and covered a wide age and disease severity range. Moreover, we also investigated lower limb muscle behaviour, which has never been assessed before with this aim.

We found no significant clinically relevant difference in intrinsic hand muscle strength between the dominant and nondominant side, either in individual muscles (FDI and APB) or their combined strength (HS). Furthermore, in $87.4 \%$ of patients, FDI and APB muscles of both sides had the same MRC score, suggesting that HS is largely symmetric at all degrees of impairment. These findings are in agreement with previous results by van Pomeren $e t a l^{6}$ and Videler $e t a l^{7}$ in smaller series, and argue strongly against the occurrence of OW in CMT1A.

Vinci et $a l^{12}$ speculated that in healthy subjects, the $\mathrm{DH}$ is stronger than the non-dominant and therefore the finding of equal strength in both hands of CMT patients would be indicative of the OW phenomenon as a consequence of greater weakening of an originally stronger $\mathrm{DH}$.

We believe that the MRC scale is not designed to pick up differences between sides in normal subjects, as a score of 5 is normal by definition, independently from the evaluated side. In this respect, a quantitative assessment of strength with a myometer would be helpful, although it cannot measure strength in intrinsic hand muscles when the MRC grade is lower than $3 .^{813}$ However, even by using a myometer specific for the hand it remains controversial whether the $\mathrm{DH}$ of healthy subjects is stronger than the NDH. Incel et $a l^{14}$ and Armstrong and Oldham $^{15}$ found that the DH was significantly stronger in righthanded subjects, but no significant difference between sides could be documented for left-handed people. In another study, Shyamal and Arvinder reported in young men and women that the $\mathrm{DH}$ was stronger than NDH both in right- and left-handed subjects. ${ }^{16}$ However, Tanaka and collaborators, by studying the electrically evoked and voluntary contractile properties of FDI from 10 healthy subjects, showed that the maximal voluntary contraction strength and maximal twitch tension were not significantly different between dominant and non-dominant sides. ${ }^{17}$ 
A second important controversial issue is whether older or more severely affected patients have further weakness due to OW. Videler $e t a l^{7}$ found a significant asymmetry in favour of $\mathrm{NDH}$ in the subgroup of 22 CMT1A patients with more severe impairment, a finding similar to that shown by CMTX1 patients with more severe weakness in the series of Arthur-Farraj et al. ${ }^{8}$ We analysed HS differences between sides in relation to age and disease severity. As expected, with increasing age and disease severity (as assessed by the CMTNS), FDI and ABP strength progressively decreased, but there were no differences between the two sides, neither with ageing nor with progression of disease severity. These data fit with those of van Pomeren and coauthors $^{6}$ and are a strong piece of evidence against the presence of OW. Interestingly, Burns et al ${ }^{18}$ showed in a large homogeneous group of CMT1A children that grip and pinch strength develop symmetrically over time, indicating no OW phenomenon in CMT1A with growth. A possible explanation of the findings of Arthur-Farraj et $a l^{8}$ on greater weakness of the $\mathrm{DH}$ in CMTX1 particularly for the median nerve innervated muscles is that CMTX1 neuropathy is non-homogeneous and asymmetric, as for a patchy nerve trunk involvement, and $\mathrm{DH}$ nerves might be more prone to chronic microtraumatic injury.

None of the previous studies considered lower limbs in order to clarify the OW hypothesis. It is debated whether a dominance effect on strength exists in the lower limbs. Sadeghi et a $l^{9}$ reported gait asymmetry in some temporal and kinematic parameters in normal subjects. Asymmetry of plantar flexor strength ${ }^{19}$ and electromyographic amplitude profiles in the soleus muscle ${ }^{20}$ in favour of the dominant side were found in other studies.

In our series, AT and TS strength was largely symmetric (in $81.1 \%$ and $94.4 \%$ of cases, respectively) and no difference between sides was found in MRC score means, either when considering individual muscles (AT and TS) or their combined strength (FS). Notably, by evaluating the effect of ageing and increasing disease severity, no difference was found for AT strength, while the dominant TS became stronger than the non-dominant.

This finding might suggest that the effect of dominance over decades, by a greater amount of daily activity, reinforces (rather than weakens) the dominant TS muscle and may partially prevent progressive weakening. TS differs in composition from AT and intrinsic hand muscles because it is a large muscle with about $80 \%$ of slowly contracting type I muscle fibres. ${ }^{21}$ Type I fibres have predominant tonic activity with high resistance and become hypertrophic after exercise, a difference from type II fibres, which have phasic activity and develop fatigue more easily. Chetlin et $a l^{22}$ reported that only type I fibres became hypertrophic after physiological exercise in CMT1A. They showed that a moderate-intensity resistance training programme in a home-based setting significantly improved strength in upper and lower limb muscles of CMT1A patients. They concluded that strengthening resulted from muscle adaptation to the resistance training due to hypertrophy of type I fibres. Notably, the diameter of type I fibres significantly correlated with total training load. ${ }^{22}$ Similarly, El Mhandi and colleagues reported that a specific training exercise programme performed three times per week improved muscle strength by producing muscle fibre hypertrophy. ${ }^{23}$ These results show that both home-based exercise and a specific rehabilitation programme are useful in maintaining muscle strength in CMT patients and are able to increase type I muscle fibre diameter. Therefore, we hypothesise that the prevalence of greater dominant than non-dominant TS strength in older and more severely affected CMT patients may be a consequence of relative overuse over decades in a muscle with type I fibre predominance.
In conclusion, all our data contradict the hypothesis of OW. A particularly strong point is that we found no effect of OW over time resulting in greater weakness in dominant muscles with increasing age or in more severely affected patients. In CMT1A, weakness is due to the disease itself and OW produces no further weakness of overloaded muscles; therefore, CMT patients should not limit the use of their limbs in daily life in order to prevent muscle strength loss because OW plays no role in disease progression.

A fundamental consequence of these results is that exercise is not harmful for CMT1A patients, and possibly for the overall CMT population. Here, we provide data for CMT1A and we cannot be certain that our conclusions apply to all the other CMT types including CMTX $1 .^{8}$

Similarly, we cannot exclude that supramaximal exercise might be detrimental in CMT and indeed most authors recommend performing aerobic exercises at a submaximal work level. ${ }^{24-26}$ For instance, Maggi et $a l^{25}$ did not find evidence of OW in their small series of patients such treated and proposed to repeat treatment at least every 6 months to prevent the loss of benefit they observed to occur. Accordingly, physical activity should be encouraged in CMT1A (and possibly in CMT as a whole), and to date rehabilitation remains the most effective treatment for this condition. ${ }^{24-26}$

\section{Author affiliations}

${ }^{1}$ IRCCS Foundation, C. Besta Neurological Institute, Milan, Italy

${ }^{2}$ MRC Centre for Neuromuscular diseases, UCL Institute of Neurology, London, UK

${ }^{3}$ Department of Neurology, Ophthalmology and Genetics, University of Genoa,

Genoa, Italy

${ }^{4}$ Department of Neurological, Neuropsychological, Morphological and Motor

Sciences, University of Verona, Verona, Italy

${ }^{5}$ Federico II University Department of Neurological Sciences, Naples, Italy

${ }^{6}$ Department of Neurosciences, University of Messina, and Clinical Centre NEMO

SUD, Fondazione Aurora Onlus, Messina, Italy

${ }^{7}$ Neurology Clinic, Magna Graecia University, and Neuroimaging Research Unit,

National Research Council, Catanzaro, Italy

${ }^{8}$ Department of Neurosciences, Catholic University and Don Gnocchi Foundation,

Rome, Italy

${ }^{9}$ Department of Neurosciences, University of Parma, Parma, Italy

${ }^{10}$ Department of Pharmacological Sciences, University School of Pharmacy, Milan, Italy

${ }^{11}$ IMDEA-Food, Madrid, Spain

${ }^{12}$ Department of Epidemiology and Biostatistics, European Institute of Oncology, Milan, Italy

Collaborators The CMT-TRIAAL Group: IRCCS Foundation, C. Besta Neurological Institute, Milan: C Marchesi, E Salsano, L Nanetti, C Marelli, V Scaioli, C Ciano, M Rimoldi, G Lauria, E Rizzetto, F Camozzi; Department of Neurology,

Ophthalmology and Genetics, University of Genoa, Genoa: E Narciso, M Grandis, M Monti-Bragadin, L Nobbio; Department of Neurological, Neuropsychological, Morphological and Motor Sciences, University of Verona, Verona: A Casano, L Bertolasi, I Cabrini, K Corrà, N Rizzuto; Department of Neurological Sciences, Federico II University of Naples, Naples: F Manganelli, C Pisciotta; Department of Neurology, "Salvatore Maugeri" Foundation, IRCCS, Telese Terme: M Nolano; Department of Neurosciences, University of Messina, Messina: A Mazzeo, R Di Leo, G Majorana, M Russo; Magna Graecia University, Neurology Clinic, and Neuroimaging Research Unit, National Research Council, Catanzaro: P Valentino, R Nisticò, D Pirritano, A Lucisano, M Canino; Institute of Neurology, Department of Neurosciences, Sacro Cuore Catholic University, and Don Gnocchi Foundation, Rome: C Pazzaglia, G Granata, M Foschini; Department of Neurosciences, University of Parma, Parma: F Brindani, F Vitetta; I Allegri; Department of Pharmacological Sciences, School of Pharmacy, University of Milan, Milan: P Bogani. (all in Italy); The CMT-TRAUK group: Medical Research Council Centre for Neuromuscular Diseases, University College London Institute of Neurology, London, UK: M Laurà, J Blake, M Koltzenburg, E Hutton, M Lunn.

Contributors DP and GP: gave substantial contributions to the conception of the work, analysis and interpretation of data and to draft the work and revise it critically for important intellectual content and gave final approval of the version to be published and agree to be accountable for all aspects of the work in ensuring that questions related to the accuracy of any part of the work are appropriately investigated and resolved. AS and DR gave substantial contributions to analysis and 
interpretation of data. RACH, MMR and AS critically revised the work for important intellectual content. All other authors gave substantial contributions to the acquisition of data for the work and gave final approval of the version to be published.

Funding This work was supported by Telethon-UILDM (grant numbers GUP04002 and GUP05007) and AIFA (Italian Medicines Agency; grant number FARM53APAH) in Italy, and by the Muscular Dystrophy Campaign in the UK (grant number RA3/736/1).

Competing interests None.

Patient consent Obtained.

Ethics approval Ethics committees of all the centres involved in the Italian/UK trial of ascorbic acid (CMT-TRIAAL/CMT-TRAUK).

Provenance and peer review Not commissioned; externally peer reviewed.

\section{REFERENCES}

1 Chan KM, Amirjani N, Sumrain M, et al. Randomized controlled trial of strength training in post-polio patients. Muscle Nerve 2003;27:332-8.

2 Johnson EW, Braddom R. Over-work weakness in facioscapulohumeral muscular dystrophy. Arch Phys Med Rehabil 1971;52:333-6.

3 Bonsett RW. Pseudohypertrophic muscular dystrophy: distribution of degenerative feature as revealed by anatomical study. Neurology 1963:13:728-38.

4 Janiszewski DW, Caroscio JT, Wisham LH. Amyotrophic lateral sclerosis: a comprehensive rehabilitation approach. Arch Phys Med Rehabil 1983;64:304-7.

5 Vinci P, Esposito C, Perelli SL, et al. Overwork weakness in Charcot-Marie-Tooth disease. Arch Phys Med Rehabil 2003;84:825-7.

6 Van Pomeren M, Selles RW, van Ginneken BT, et al. The hypothesis of overwork weakness in Charcot-Marie-Tooth: a critical evaluation. J Rehabil Med 2009;41:32-4.

7 Videler AJ, Beelen A, Nollet F. Verifying the hypothesis of overwork weakness in Charcot-Marie-Tooth. J Rehabil Med 2010;42:380.

8 Arthur-Farraj PJ, Murphy SM, Laura M, et al. Hand weakness in Charcot-Marie-Tooth disease 1X. Neuromuscul Disord 2012;22:622-6.

9 Sadeghi $H$, Allard $P$, Prince $F$, et al. Symmetry and limb dominance in able-bodied gait: a review. Gait Posture 2000;12:34-45.

10 Pareyson D, Reilly MM, Schenone A, et al. Ascorbic acid in Charcot-Marie-Tooth disease type 1A (CMT-TRIAAL and CMT-TRAUK): a double-blind randomised trial. Lancet Neurol 2011;10:320-8.
11 Videler AJ, Beelen A, van Schaik IN, et al. Tripod pinch strength and thumb opposition are the major determinants of manual dexterity in Charcot-Marie-Tooth disease type 1A. J Neurol Neurosurg Psychiatry 2010;81:828-33.

12 Vinci P, Perelli SL, Gargiulo P. About the hypothesis of overwork weakness in Charcot-Marie-Tooth disease. J Rehabil Med 2009:41:778.

13 Selles RW, van Ginneken BT, Schreuders TA, et al. Dynamometry of intrinsic hand muscles in patients with Charcot-Marie-Tooth disease. Neurology 2006:67:2022-7.

14 Incel NA, Ceceli E, Durukan PB, et al. Grip strength: effect of hand dominance. Singapore Med J 2002;43:234-7.

15 Armstrong CA, Oldham JA. A comparison of dominant and non-dominant hand strengths. J Hand Surg Br 1999:24:421-5.

16 Shyamal K, Arvinder PS. Effect of hand dominance in grip strength in collegiate population of Amritsar, Punjab, India. Anthropologist 2010;12:13-16.

17 Tanaka M, McDonagh MJ, Devies CT. A comparison of the mechanical proprieties of the first dorsal interosseous in the dominant and non dominant hand. Eur J Appl Physiol Occup Physiol 1984;53:17-20.

18 Burns J, Bray P, Cross LA, et al. Hand involvement in children with Charcot-Marie-Tooth disease type 1A. Neuromuscul Disord 2008;18:970-3.

19 Damholt V, Termansen NB. Asymmetry of planter flexion strength in the foot. Acta Orthop Scand 1978:49:215-19.

20 Arsenault $A B$, Winter DA, Marteniuk RG. Is there a normal profile of EMG activity in gait. Med Biol Eng Comput 1986;24:337-43.

21 Gollnick PD, Sjödin B, Karlsson J, et al. Human soleus muscle: a comparison of fiber composition and enzyme activities with other leg muscles. Pflugers Arch 1974;348:247-55.

22 Chetlin RD, Gutmann L, Tarnopolsky M, et al. Resistance training effectiveness in patients with Charcot-Marie-Tooth disease: recommendations for exercise prescription. Arch Phys Med Rehabil 2004;85:1217-23.

23 El Mhandi L, Millet GY, Calmels $P$, et al. Benefits of interval-training on fatigue and functional capacities in Charcot-Marie-Tooth disease. Muscle Nerve 2008;37:601-10.

24 White CM, Pritchard J, Turner-Stokes L. Exercise for people with peripheral neuropathy. Cochrane Database Syst Rev 2004;(4):CD003904.

25 Maggi G, Monti Bragadin M, Padua L, et al. Outcome measures and rehabilitation treatment in patients affected by Charcot-Marie-Tooth neuropathy: a pilot study. Am J Phys Med Rehabil 2011;90:628-37.

26 Lindeman $E$, Leffers $P$, Spaans $F$, et al. Strength training in patients with myotonic dystrophy and hereditary motor and sensory neuropathy: a randomized clinical trial. Arch Phys Med Rehabil 1995;76:612-20. 